\title{
CORRELAÇÃO ENTRE OBSTRUÇÃO ARTERIAL PERIFÉRICA E OBSTRUÇÃO DE VIAS AÉREAS EM PORTADORES DE DPOC NÃO REABILITADOS
}

\author{
Diogo Fanfa Bordin ${ }^{1}$ \\ Ricardo Gass ${ }^{2}$ \\ Cássia da Luz Goulart ${ }^{3}$ \\ Dannuey Machado Cardoso ${ }^{4}$ \\ Lisiane Lisboa Carvalho ${ }^{4}$ \\ Andrea Lúcia Gonçalves da Silva ${ }^{4}$ \\ Dulciane Nunes Paiva
}

\section{RESUMO}

A Doença Pulmonar Obstrutiva Crônica (DPOC) produz desordens fisiopatológicas respiratórias com severo comprometimento dos volumes pulmonares, podendo também ocasionar alterações extrapulmonares como as doenças arteriais periféricas (DAP). O estudo objetivou correlacionar o Índice Tornozelo-Braquial (ITB) com o Volume Expiratório Forçado no Primeiro Segundo $\left(\mathrm{VEF}_{1}\right)$ e Fluxo Expiratório Forçado entre $25 \%$ e $75 \%$ da Capacidade Vital Forçada $\left(\mathrm{FEF}_{25-75 \%}\right)$ em portadores de DPOC. Trata-se de um estudo correlacional com amostra selecionada por conveniência. Foram avaliados portadores de DPOC com estadiamentos Global Initiative for Chronic Obstructive Lung Disease (GOLD) entre II e IV. O ITB foi avaliado em membros superiores e membros inferiores através de esfigmomanômetro e doppler vascular portátil. A função pulmonar foi avaliada por espirometria digital, obtendo-se as variáveis capacidade vital forçada (CVF), $\mathrm{VEF}_{1}$ e $\mathrm{FEF}_{25}$ $75 \%$. Foram avaliados 12 indivíduos com média de idade de 66,58 $\pm 6,1$ anos e com Índice de Massa Corpórea de $29,21 \pm 6,7 \mathrm{Kg} / \mathrm{m}^{2}$. Houve predominância do sexo masculino ( $\mathrm{n}=8,66,7 \%$ ).

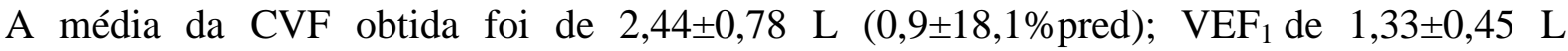
(50,4 $\pm 15,5 \%$ pred), $\mathrm{VEF}_{25-75 \%}$ de $0,65 \pm 0,32 \mathrm{~L}(26,9 \pm 16,6 \%$ pred) e do ITB de $0,88 \pm 0,12$. Foi evidenciada correlação positiva moderada entre $\operatorname{ITB}_{\text {e }} \operatorname{VEF}_{1}(r=0,684 ; p=0,014)$. Nos portadores de DPOC avaliados, a presença de obstrução de via aérea alta apresentou-se relacionada à presença de doença arterial periférica.

Palavras-chave: DPOC. Doenças vasculares. Função pulmonar.

\footnotetext{
${ }^{1}$ Fisioterapeuta pela Universidade de Santa Cruz do Sul, RS. Residente em Urgência e Emergência, Hospital de

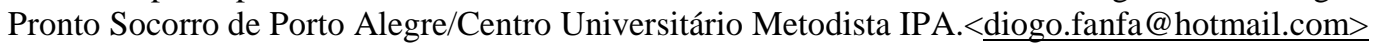

${ }^{2}$ Fisioterapeuta pela Universidade de Santa Cruz do Sul (UNISC), Santa Cruz do Sul - RS. Mestrando em Ciências Pneumológicas pela Universidade Federal do Rio Grande do Sul (UFRGS). <ricardogass@ hotmail.com>

${ }^{3}$ Aluna do Curso de Fisioterapia da Universidade de Santa Cruz do Sul. $<$ luz.cassia@hotmail.com>

4 Professores do Departamento de Educação Física e Saúde na Universidade de Santa Cruz do Sul.<dannueyc@unisc.br><lisianecarvalho@unisc.br><andreag@unisc.br>

5 Professora do Departamento de Educação Física e Saúde e do Programa de Pós-Graduação em Promoção da Saúde (PPGPS) na Universidade de Santa Cruz do Sul. < $\underline{\text { dulciane@unisc.br> }}$
} 


\begin{abstract}
Chronic Obstructive Pulmonary Disease (COPD) produces respiratory pathophysiological disorders with severe impairment in lung volume and can also cause extrapulmonary disorders such as peripheral arterial disease (PAD). The study aimed relate the Ankle-Brachial Index $(\mathrm{ABI})$ with the forced expiratory volume in one second $\left(\mathrm{FEV}_{1}\right)$ and forced expiratory flow between $25 \%$ and $75 \%$ of forced vital capacity (FEF 25-75\%) in patients with COPD. This is a correlational study with a sample selected by convenience. Were evaluated patients with COPD staging Global Initiative for Chronic Obstructive Lung Disease (GOLD) between II and IV. The ABI was measured in upper limbs and lower limbs through sphygmomanometer and Doppler vascular laptop. Pulmonary function was assessed by spirometry digital, obtaining the variables forced vital capacity (FVC), $\mathrm{FEV}_{1}$ and $\mathrm{FEF} 25-75 \%$. They evaluated 12 individuals with mean age of $66.58 \pm 6.1$ years, and body mass index of $29.21 \pm 6.7 \mathrm{~kg} / \mathrm{m}^{2}$.There was a predominance of males $(\mathrm{n}=8,66.7 \%)$. Mean FVC obtained $2.44 \pm 0.78 \mathrm{~L}(0.9 \pm 18,1 \%$ pred $) ; \mathrm{FEV}_{1}$ of $1.33 \pm 0.45 \mathrm{~L}(50.4 \pm 15.5 \%$ pred $), \mathrm{VEF}_{25-75 \%} 0.65$ $\pm 0.32 \mathrm{~L}(26.9 \pm 16.6 \%$ pred $)$. Average ABI of $0.88 \pm 0.12$. Evidenced moderate positive correlation between $\mathrm{ABI}$ and $\mathrm{FEV}_{1}(\mathrm{r}=0.684 ; \mathrm{p}=0.014)$. In patients with COPD evaluated, the presence of upper airway obstruction had to be related to the presence of peripheral arterial disease.
\end{abstract}

Keywords: COPD. Vascular diseases. Pulmonary function.

\title{
1 INTRODUÇÃO
}

Sabe-se que o principal fator para o surgimento da Doença Pulmonar Obstrutiva Crônica (DPOC) é o tabagismo, devendo ser ressaltado que $80 \%$ a $90 \%$ dos indivíduos portadores desta doença são tabagistas. Ressalta-se,entretanto, que apenas $10 \%$ a $20 \%$ destes desenvolvem a patologia (CHENG et al., 2009; YOSHIDA e TUDER, 2007). O tabagismo também é considerado o principal fator de risco prevenível para várias doenças crônicas, entre elas, as doenças arteriais periféricas (DAP) (SILVA et al., 2009).

Neste sentido, o levantamento da história e da carga tabágica nos portadores de DPOC é fundamental, visto que além da própria patologia, o tabagismo também induz a um processo inflamatório crônico-induzido no tecido pulmonar e posteriormente à inflamação sistêmica. Além disso, o ato tabágico provoca dano endotelial vascular, via estresse oxidativo, que associados aos fatores acima descritos podem contribuir para o desenvolvimento de processos ateroscleróticos (LIN et al., 2013).

O Índice Tornozelo Braquial (ITB) se refere a um dos parâmetros utilizados para avaliar a menor perfusão arterial e assim diagnosticar DAP. O mesmo pode ser definido como a razão entre a pressão sistólica dos membros inferiores (artérias pediosa e tibial posterior) e dos membros superiores (artéria braquial), podendo ser utilizado para avaliar alterações 
vasculares estruturais e funcionais para identificar o desempenho hemodinâmico do leito vascular arterial e definir a sua gravidade (RESNICK et al., 2004).

Tendo em vista que o tabagismo é um fator de risco em comum para DAP, considera-se importante avaliar a presença de doenças arteriais periféricas em portadores de DPOC. Dessa forma, o presente estudo objetivou relacionar o Índice Tornozelo-Braquial com os volumes pulmonares deportadores de DPOC ingressantes em um Programa de Reabilitação Pulmonar.

\section{FUNDAMENTAÇÃO TEÓRICA}

A DPOC é definida como uma doença respiratória prevenível e tratável, caracterizada por obstrução crônica ao fluxo aéreo, não sendo totalmente reversível, produzindo também desordens fisiopatológicas respiratórias com severo comprometimento dos volumes pulmonares (TREVISAN e PORTO, 2010) e também ocasionando alterações extrapulmonares diretas ou indiretas ao ato tabágico (SOCIEDADE BRASILEIRA DE PNEUMOLOGIA E TISIOLOGIA, 2004). Considerado como um dos principais fatores de risco para o desenvolvimento de DAP, o tabagismo é o principal causador da DPOC (SOARES et al., 2014; CRIQUI, 2001).

Com o mesmo fator de risco causal e presença de inflamação sistêmica crônica, a doença cardiovascular (DCV) se encontra como umas das principais causadoras de morte do mundo. Conforme relatado pela Organização Mundial de Saúde (LIN; ZHANG; ZHANG; 2013), as DCV são doenças crônicas que ocorrem por efeitos acumulativos em longo prazo dos fatores de risco e continuarão a dominar as tendências da mortalidade nas próximas décadas (LIN et al., 2013). Existem algumas vias comuns que podem ligar a DPOC à DCV, tais como a inflamação crônica, com o aumento dos níveis do fator de necrose tumoral alfa (TNF- $\alpha$ ), interleucina 6 e 8 e proteína $\mathrm{C}$ reativa. As doenças vasculares periféricas são prevalentes em portadores de DPOC e se estima que a redução de $10 \%$ no volume expiratório forçado no primeiro segundo $\left(\mathrm{VEF}_{1}\right)$ esteja associada a $30 \%$ de aumento do risco de morte cardiovascular (BLUM, SIMSOLO e SIRCHAN, 2013; LIN et al., 2013).

No conjunto das DCV está a doença arterial periférica (DAP). Apesar de apresentar variação ampla ao redor do mundo, a DAP tem uma alta prevalência entre portadores de DPOC (LIN et al., 2013). A mesma se manifesta de forma assintomática, estando associada a alto risco cardiovascular (ALZAMORA et al., 2013). Um estudo realizado na França (CASTAGNA et al., 2008) constatou a presença elevada de DAP, em 81,4\% dos portadores 
de DPOC, quando comparada aos 30 a $40 \%$ em estudos realizados em Israel (BLUM et al., 2011) e Espanha (PECCI et al., 2012), respectivamente. No entanto, até o momento não há estudos publicados sobre a prevalência de DAP em portadores de DPOC no Brasil (LIN et al., 2013). Ainda, segundo Lin et al. (2013) não foi encontrada diferença significativa ao se comparar os estadiamentos da DPOC com presença ou não de DAP (LIN et al., 2013).

A doença cardiovascular (DCV) coexistente na DPOC caracteriza-se pela presença de inflamação sistêmica crônica, sendo uma das principais causas de morte no mundo (LIN et $a l ., 2013)$ e a DAP pode ser definida como estreitamento e/ou enrijecimento do lúmen arterial nos membros inferiores, como parte do processo aterosclerótico vascular sistêmico (MAKDISSE et al., 2007). A claudicação intermitente é o sintoma clássico e caracteriza-se pela ocorrência de dor ao esforço no membro acometido, o que pode restringir as atividades de vida diária do paciente. Deve ser ressaltado que associado à claudicação, ocorrem alterações pressóricas no membro inferior acometido (ABOYANS et al., 2012).

Neste aspecto, os parâmetros fisiológicos do coração e artérias podem ser medidos a partir da superfície do corpo, por meio de testes não-invasivos e podem ser utilizados para a identificação de mecanismos multi-físicos do sistema cardiovascular, dentre eles a pressão arterial (PA) (LIN; ZHANG; ZHANG; 2013). O ITB tornou-se útil em avaliar alterações estruturais e funcionais do vaso sanguíneo, sendo utilizado para identificar doenças vasculares periféricas (RESNICK et al.,2004).

Neste sentido, hipotetiza-se que portadores de DPOC não participantes de um Programa de Reabilitação Pulmonar possam apresentar alta prevalência de DAP e que esta pode estar associada à obstrução de vias aéreas.

\section{MATERIAIS E MÉTODOS}

O presente estudo abrange uma investigação correlacional com amostra por conveniência em andamento, composto por indivíduos com diagnóstico clínico de DPOC e estadiamento II a IV, segundo critério da Global Initiative for Lung Disease (GOLD), tendo sido aprovado pelo Comitê de Ética e Pesquisa com Seres Humanos sob protocolo $\mathrm{n}^{\circ}$ 22806913.8.0000.5343 e registrado no Clinical Trials sob protocolo $\mathrm{n}^{\mathrm{o}} 435093 / 2013$. Todos os indivíduos submetidos ao estudo assinaram o Termo de Consentimento Livre e Esclarecido. A amostra avaliada faz parte do Programa de Reabilitação Pulmonar (RP) do Hospital Santa Cruz, Santa Cruz do Sul - RS, Brasil, onde foram avaliados portadores de 
DPOC não reabilitados, ingressantes no Programa de Reabilitação Pulmonar do Hospital Santa Cruz (HSC), Santa Cruz do Sul - RS. Foram incluídos indivíduos com idade entre 40 a 80 anos, com prova espirométrica para diagnóstico de DPOC com estadiamentos proposto pelaGOLDentre II e IV e estáveis clinicamente. Segundo GOLD (2014), os pacientes podem ser classificados em 04estadiamentos, onde GOLD I = grau leve $\left(\mathrm{VEF}_{1}<80 \%\right.$ predito); GOLD II = grau moderado $\left(50 \% \leq \mathrm{VEF}_{1}<80 \%\right.$ predito $)$, GOLD III = grau severo $(30 \% \leq$ $\mathrm{VEF}_{1}<50 \%$ predito $)$ e GOLD IV = grau muito severo $\left(\mathrm{VEF}_{1}<30 \%\right.$ predito $)$.

Foram excluídos aqueles que apresentassem desordens musculoesqueléticas, câncer de pulmão, déficits cognitivos, lesões cutâneas na região dos pés e tornozelos e agudização da doença em 30 dias prévios ao estudo. Inicialmente, os indivíduos foram avaliados quanto às suas características antropométricas, sendo elas peso, altura e índice de massa corpórea (IMC). Para classificação do IMC foram utilizados valores específicos para DPOC, considerando valores $<22 \mathrm{Kg} / \mathrm{m}^{2}$ como magreza, de $22-27 \mathrm{Kg} / \mathrm{m}^{2}$ como eutrófico e $>27 \mathrm{Kg} / \mathrm{m}^{2}$ como obesidade (CUPPARI, 2002). Após, os indivíduos foram avaliados quanto ao ITB e função pulmonar.

Para se obter a classificação do estadiamento da DPOC foi realizada avaliação espirométrica (EasyOne ${ }^{\circledR}$, Modelo 2001, Suíça), visando a avaliação da capacidade vital forçada (CVF), do volume expirado forçado no primeiro segundo $\left(\mathrm{VEF}_{1}\right)$, do fluxo expirado forçado entre $25 \%$ e $75 \%$ da capacidade vital $\left(\mathrm{FEF}_{25 \%-75 \%}\right)$ e da relação $\mathrm{VEF}_{1} / \mathrm{CVF}_{\%}$, sendo seus valores expressados de acordo com o predito e conforme as diretrizes da American Thoracic Society/European Respiratory Society (2002).

O ITB foi aferido com os indivíduos em decúbito dorsal conforme recomendação da American Heart Association (ABOYANS et al., 2012). A aferição da PAS das artérias braquiais, pediosa e tibial posterior bilateralmente foi realizada através de doppler vascular

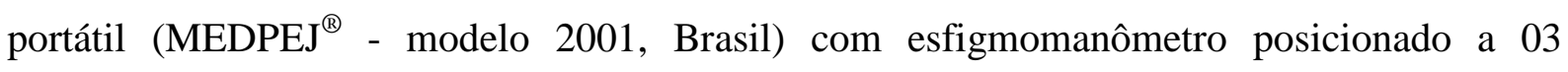
centímetros acima da fossa cubital nos membros superiores (MS) e 03 centímetros acima do maléolo medial em membros inferiores (MI). Após as medidas, dividiu-se o maior valor entre os MI (PAS $\mathrm{mi}_{\text {i }}$ ) pelo maior valor entre os membros superiores $\left(\mathrm{PAS}_{\mathrm{ms}}\right)$ para o cálculo do ITB geral $\left(\mathrm{ITB}_{\text {geral }}=\mathrm{PAS}_{\mathrm{mi}} / \mathrm{PAS}_{\mathrm{ms}}\right.$ ) (ABOYANS et al., 2012). Os portadores de DPOC avaliados foram classificados conforme Kim, Wattanakit e Gornik (2012), sendo que um índice de 1.00 a 1.40 é considerado normal, 0.91 a 0.99 limítrofe e menores que 0.90, DAP.

Os dados foram tabulados e analisados através do software Statistical Package for the Social Sciences (SPSS, v.20.0). A normalidade dos dados foi avaliada pelo teste de Shapiro-Wilk, sendo posteriormente expressos em média \pm desvio padrão e distribuição de 
frequências. A correlação dos dados foi analisada com o teste de Spearman, com nível de significância com $\mathrm{p}<0,05$.

\section{RESULTADOS E DISCUSSÃO}

Foram avaliados 12 pacientes, sendo $08(66,7 \%)$ do sexo masculino, cuja média de idade dos pacientes revelou uma amostra idosa e com média elevadade IMC na faixa de obesidade (Tabela 1).

A predominância de portadores de DPOC do sexo masculino apresentado em nossos resultados foi também constatada por Crestani et al. (2010) que desenvolveu estudo na cidade de Santa Cruz do Sul - RS no período de 2007 a 2008 que avaliou o perfil de mortalidade por DPOC, a ocorrência de 1987 mortes, excluindo-se os óbitos fetais. Deste total, 113 ocorreram por DPOC, com uma prevalência de 5,68\%. Quando analisados os sexos, constatou-se um número de 74 óbitos do sexo masculino e 39 do sexo feminino $(65,48 \%$ e $34,52 \%$ respectivamente).

A média de idade avançada dos pacientes também é outra característica da doença, visto que o surgimento dos sintomas surge tardiamente devido ao caráter crônico e progressivo da mesma (GODOY et al., 2007). 
Tabela 1. Características antropométricas e de função pulmonar dos indivíduos analisados.

\begin{tabular}{|c|c|}
\hline Variáveis & $n=12$ \\
\hline \multicolumn{2}{|c|}{ Características antropométricas } \\
\hline Idade (anos) & $66,58 \pm 6,14$ \\
\hline Sexo masculino, n (\%) & $8(66,7)$ \\
\hline $\operatorname{IMC}\left(\mathrm{Kg} / \mathrm{m}^{2}\right)$ & $29,21 \pm 6,73$ \\
\hline Magreza, n (\%) & $1(8,3)$ \\
\hline Eutrófico, n (\%) & $4(33,3)$ \\
\hline Obesidade, n (\%) & $7(58)$ \\
\hline \multicolumn{2}{|l|}{ Função pulmonar } \\
\hline CVF (L) & $2,44 \pm 0,78$ \\
\hline $\mathrm{CVF} \%$ & $70,95 \pm 18,18$ \\
\hline $\mathrm{VEF}_{1}(\mathrm{~L})$ & $1,33 \pm 0,45$ \\
\hline $\mathrm{VEF}_{1} \%$ & $50,45 \pm 15,53$ \\
\hline $\mathrm{VEF}_{25-75}(\mathrm{~L})$ & $0,65 \pm 0,32$ \\
\hline $\mathrm{VEF}_{25-75} \%$ & $26,90 \pm 16,64$ \\
\hline \multicolumn{2}{|l|}{ Estadiamento GOLD } \\
\hline II, n (\%) & $6(50)$ \\
\hline III, n $(\%)$ & $5(41,7)$ \\
\hline $\mathrm{IV}, \mathrm{n}(\%)$ & $1(8,3)$ \\
\hline
\end{tabular}

De acordo com os valores espirométricos obtidos, pode-se observar uma amostra com padrão obstrutivo e restritivo clássico da DPOC. A análise da média do ITB geral dos pacientes apresentou valores reduzidos, demonstrando alta ocorrência de DAP. A Tabela 2 apresenta as estratificações dos valores conforme suas classificações. Cabe ressaltar que tais pacientes nunca participaram de programas de RP, sendo estes pacientes ingressantes no referido programa. Conforme demonstrado na Tabela 1, os pacientes avaliados apresentam grande comprometimento pulmonar, o que segundo Paulin et al. (2003) e Sudbrack e Leite (2007), faz com que o indivíduo apresente redução importante em seu desempenho físico, devido a fatores como hiperinsuflação dinâmica e aumento do metabolismo muscular glicolítico, levando-os à inatividade e ao sedentarismo, fatores de risco para surgimento de DAP, além do próprio tabagismo (SILVA et al., 2009), o que pode justificar nossos achados, onde encontramos presença de DAP. Silva et al. (2012) demonstraram alta relação entre arteriopatias e sobrepeso, o que vem ao encontro aos resultados apresentados no presente estudo. 
Tabela 2. Análise e estratificação do Índice Tornozelo-Braquial dos indivíduos analisados.

\begin{tabular}{lcc}
\hline Variáveis & $\mathbf{n = 1 2}$ & $\mathbf{n}(\boldsymbol{\%})$ \\
\hline ITB geral & & $0,88 \pm 0,12$ \\
DAP & $0,76 \pm 0,06$ & $5(41,7)$ \\
Limítrofe & $0,94 \pm 0,03$ & $5(41,7)$ \\
Normal & $1,04 \pm 0,05$ & $2(16,7)$ \\
\hline
\end{tabular}

$\mathrm{ITB}=$ índice tornozelo-braquial; $\mathrm{DAP}=$ doença arterial periférica. Dados expressos em média \pm desvio padrão e distribuição de frequência.

Houve correlação positiva de poder estatístico moderado entre $\mathrm{VEF}_{1}$ e ITB geral, não ocorrendo o mesmo quando analisado $\operatorname{VEF}_{25-75 \%}(\mathrm{r}=0,240 ; \mathrm{p}=0,452)$ e ITB geral (Figura 1), podendo ser constatado então que, na amostra avaliada, quanto maior os valores preditos de $\mathrm{VEF}_{1}$, ou seja, menor obstrução de vias aérea alta, maior também foram os valores de ITB dos pacientes. Tal dado pode inferir relação entre a gravidade da DPOC expressa pelo $\mathrm{VEF}_{1}$ e a presença de doença arterial periférica.

Figura 1. Correlação entre Volume Expiratório Forçado no primeiro segundo $\left(\mathrm{VEF}_{1}\right)$ e Índice Tornozelo-Braquial (ITB) geral.

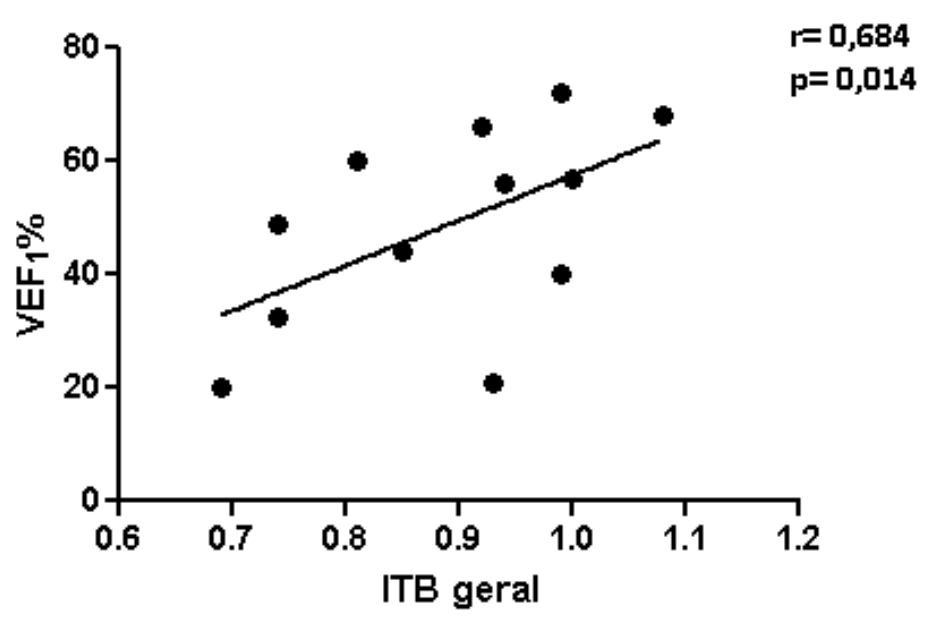

Teste de correlação de Spearman. Nível de significância com um p $<0,05$.

Devem ainda serem consideradas as limitações presentes no estudo como o baixo $n$ amostral, bem como o fato da doença arterial periférica não ter sido avaliada por exames de imagem que possibilitassem o diagnóstico clínico da mesma. 


\section{CONCLUSÃO}

Nos portadores de DPOC avaliados, a presença de obstrução de via aérea alta apresentou-se relacionada à presença de doença arterial periférica, sendo contatado que a maior parte da amostra era composta por indivíduos com sobrepeso. Com a perspectiva do seguimento do estudo, será avaliada a relação entre arteriopatia e sobrepeso, na tentativa de elucidar o quanto o peso corporal pode ser contributivo a ocorrência de doença arterial periférica.

\section{SUPORTE E FINANCIAMENTO}

Agradecemos a Universidade de Santa Cruz do Sul- UNISC, DECIT/SCTIEMS/FAPERGS/CNPq 1264-2551/13-1 e ao Hospital Santa Cruz.

\section{REFERÊNCIAS}

ABOYANS, V., CRIQUI, M. H., ABRAHAM, P. et al. Measurement and interpretation of the ankle-brachial index: a scientific statement from the American Heart Association. Circulation Journal of the American Heart Association, n. 24, v. 126, p. 2890-2909,nov. 2012.

ALZAMORA, M. T., FORÉS, R., PERA, G. et al. Ankle-brachial index and the incidence of cardiovascular events in the Mediterranean low cardiovascular risk population ARTPER cohort. BMC Cardiovascular Disorders, n. 119, v. 13, dez. 2013 Disponível em: <http://www.biomedcentral.com/1471-2261/13/119>.Acesso em: out. 2015.

AMERICAN THORACIC SOCIETY/EUROPEAN RESPIRATORY SOCIETY (ATS/ERS). Statement on respiratory muscle testing. American Journal of Respiratory Critical Care Medicine, n. 4, v. 166, p. 518-524,ago. 2002.

BLUM, A., SIMSOLO, C., SIRCHAN, R., HAIEK, S. "Obesity paradox" in chronic obstructive pulmonary disease. Israel Medical Association Journal, v. 13, p. 672-675, nov. 2011 .

BLUM, A., SIMSOLO, C., SIRCHAN, R. Vascular responsiveness in patients with chronic obstructive pulmonary disease (COPD). European Journal of Internal Medicine, n. 4, v. 25, p. 370-373, abr. 2013.

CASTAGNA, O., BOUSSUGES, A., NUSSBAUM, E., MARQUESTE, L., BRISSWALTER, J. Peripheral arterial disease: an underestimated aetiology of exercise intolerance in chronic obstructive pulmonary disease patients. European Journal Cardiovascular Preventive Rehabilitation, v. 15, p. 270-277, ago. 2008. 
CHENG, S.L., YU, C. J., YANG, P. C. Genetic polymorphisms of cytochrome p450 and matrix metalloproteinase in chronic obstructive pulmonary disease. Biochemical Genetics, n. 7/8, v. 47, p. 591-601, ago. 2009.

CRESTANI, G., RUTZEN, W., SILVA, A. L. G., FlEIG, T. C. M., RODRIGUES, M. T. Análise do perfil de mortalidade por Doença Pulmonar Obstrutiva Crônica no município de Santa Cruz do Sul, RS, Brasil. Revista Jovens Pesquisadores, n. 1, v. 1, p. 10-16, 2011.

CRIQUI, M. H. Peripheral arterial disease - epidemiological aspects. Vascular Medicine, Londres, n. 3, v. 6, p. 03-07, 2001.

CUPPARI, L (Coord.). Doenças Pulmonares. In: Cuppari, L. Guia de nutrição: nutrição clínica no adulto. Barueri: Manole, 2002. p. 249-62.

GOLD - Global Initiative for Chronic Obstructive Lung Disease. Global Strategy for the Diagnoses, management, and prevention of Chronic Obstructive Pulmonary Disease. Disponível em: <www.goldcopd.org>. Acesso em: 20 jul. 2014.

GODOY, I. et al. Programa de cessação de tabagismo como ferramenta para o diagnóstico precoce de Doença Pulmonar Obstrutiva Crônica. Jornal Brasileiro de Pneumologia, São Paulo, n. 2, v. 33, p. 282-286, maio/jun. 2007.

KIM, E. H., WATTANAKIT, K., GORNIK, H. L. Using the ankle-brachial index to diagnose peripheral artery disease and assess cardiovascular risk. Cleveland Clinic Journal of Medicine, n. 9, p. 79, p. 651-661, set. 2012.

LIN, M.-S., HSU, K.-Y., CHEN, Y.-J. et al. Prevalence and risk factors of asymptomatic peripheral arterial disease in patients with COPD in Taiwan. Plos One,n. 5, v. 8, maio 2013. Disponível em: 〈http://journals.plos.org/plosone/article?id=10.1371/journal.pone.0064714>. Acesso em: out. 2015.

LIN, W-H., ZHANG, H., ZHANG, Y-T. Investigation on cardiovascular risk prediction using physiological parameters. Computational and Mathematical Methods in Medicine, 2013. Disponível em: <http://www.hindawi.com/journals/cmmm/2013/272691/>. Acesso em: out. 2015.

MAKDISSE, M., NETO, R. N., CHAGAS, A. C. P. et al. Versão em português, adaptação transcultural e validação do questionário de claudicação de Edimburgo. Arquivos Brasileiros de Cardiologia, São Paulo, n. 5, v. 88, p. 501-506, maio 2007.

PAULIN, E., BRUNETTO, A. F., CARVALHO, C. R. F. Efeitos de programa de exercícios físicos direcionado ao aumento da mobilidade torácica em pacientes portadores de Doença Pulmonar Obstrutiva Crônica. Jornal Brasileiro de Pneumologia, São Paulo, n. 5, v. 29, p. 43-48, set./out.2003.

PECCI., R., AGUADO, D. F. J., SANJURJO, R. A. B., SANCHEZ, C. P., CORBACHO, A. M. Peripheral arterial disease in patients with chronic obstructive pulmonary disease. Journal of the International Union of Angiology, v. 31, n.5, p. 444-453, ago. 2012. 
RESNICK, H. E., LINDSAY, R. S. McDERMOTT, M. M. et al. Relationship of high and low ankle brachial index to all-cause and cardiovascular disease mortality: the strong heart study. Circulation, n. 6, v. 109, p. 733-739, fev.2004.

SILVA G. A., VALENTE JG, ALMEIDA LM. et al. Tabagismo e escolaridade no Brasil 2006. Revista de Saúde Pública, n. 2, v. 43, p. 48-56, ago. 2009.

SILVA, R. C. G., GIRIBELA, C. R. G., WOLOSKER, N. et al. Limitação funcional e claudicação intermitente: impacto das medidas de pressão arterial. Arquivos Brasileiros de Cardiologia, n. 2, v. 98, p. 161-166, fev.2012.

SOARES, F. dos S., JUNGES, C. de C., MURBACH, L. D., ARAÚJO, R. A. G., MACHADO, J. L. C. Prevalência de tabagismo e doença pulmonar obstrutiva crônica em doentes arteriais periféricos: resultados preliminares. FIEP Bulletin, n. 2, v. 84, 2014.

SOCIEDADE BRASILEIRA DE PNEUMOLOGIA E TISIOLOGIA. II Consenso Brasileiro sobre Tuberculose: Diretrizes Brasileiras para Tuberculose, 2004. Jornal Brasileiro de Pneumologia,São Paulo, suppl. 1, v. 30, p. 04-56, jun. 2004.

SUDBRACK, A. C., LEITE, R. S. Efetividade do exercício na claudicação. Revista Brasileirade Cardiologia Invasiva, São Paulo, n. 3, v. 15, p. 261-266, 2007.

TREVISAN, M. E.; PORTO, A. S.; PINHEIRO, T. M. Influência do treinamento da musculatura respiratória e de membros inferiores no desempenho funcional de indivíduos com DPOC. Fisioterapia e Pesquisa, São Paulo, n. 3, v. 17, p. 209-213, jul./set. 2010.

YOSHIDA, T., TUDER, R. M. Pathobiology of cigarette smoke-induced chronic obstructive pulmonary disease. Physiological Reviews, n. 3, v. 87, p. 1047-1082, jul. 2007. 